\title{
Extracellular mitochondrial DNA promote NLRP3 inflammasome activation and induce acute lung injury through TLR9 and NF-кB
}

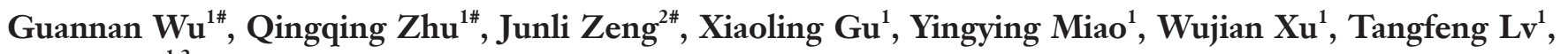 \\ Yong Song ${ }^{1,2}$ \\ ${ }^{1}$ Department of Respiratory Medicine, Jinling Hospital, Nanjing University School of Medicine, Nanjing 210002, China; ${ }^{2}$ Department of Respiratory \\ Medicine, Jinling Hospital, Southern Medical University (Guangzhou), Nanjing 210002, China \\ Contributions: (I) Conception and design: G Wu, T Lv, Y Song; (II) Administrative support: T Lv, Y Song; (III) Provision of study materials or \\ patients: G Wu, Q Zhu, J Zeng, X Gu; (IV) Collection and assembly of data: G Wu, Q Zhu, Y Miao, W Xu; (V) Data analysis and interpretation: G \\ Wu, Q Zhu, Y Song; (VI) Manuscript writing: All authors; (VII) Final approval of manuscript: All authors. \\ \#These authors contributed equally to this work. \\ Correspondence to: Yong Song, MD, PhD. 305, East Zhongshan Road, Nanjing 210002, China. Email: yong_song6310@yahoo.com.
}

Background: Extracellular mitochondrial DNA (mtDNA) was demonstrated to be capable of inducing pulmonary inflammation through TLR9 while its role in NLRP3 inflammation activation remains unknown. Methods: C57BL/6 mice were challenged intratracheally with mtDNA. Pulmonary pathology, the NLRP3 and caspase-1 p20 in lung tissues were assayed. PMA-primed THP-1 macrophages were incubated with mtDNA in vitro and cell-free medium were concentrated to detect caspase-1 p20 subunit and NLRP3 by Western blotting. Additionally, IL-1 $\beta, \mathrm{L}-18, \mathrm{TNF}-\alpha$ and caspase- 1 activity in culture were also analyzed by ELISA kits and activity assay kit.

Results: Intratracheal administration of mtDNA increased NLRP3 and caspase-1 p20 subunit in lung together with excessive inflammation and damage. Inhibition of caspase- 1 substantially diminished mtDNAinduced lung injury and inflammation. Exposed to mtDNA in THP-1 macrophages resulted in significant up-regulation of NLRP3 and increased caspase-1 p20 subunit release in culture. It also led to significant increased transcripts of NLRP3, ASC, caspase- 1 and release of IL-1 $\beta$, IL-18 and TNF- $\alpha$ in culture media. Futhermore, mtDNA exposure resulted in significant up-regulation of phosho -p38 MAPK and nucleus translocation of NF-кB. mtDNA-induced Transcripts of NLRP3 and ASC were inhibited by p38 siRNA inhibitor or NF- $\mathrm{BB}$ inhibitor.

Conclusions: Extracellular mtDNA promote NLRP3 inflammasome activation, acute pulmonary inflammation and injury through TLR9, p38 MAPK and NF- $\mathrm{B}$ pathways.

Keywords: mtDNA; NOD-like receptor family, pyrin domain containing 3 (NLRP3); acute lung injury (ALI); p38 MAPK; NF-кB

Submitted Oct 31, 2018. Accepted for publication Aug 22, 2019.

doi: $10.21037 /$ jtd.2019.10.26

View this article at: http://dx.doi.org/10.21037/jtd.2019.10.26

\section{Introduction}

Acute lung injury (ALI) and acute respiratory distress syndrome (ARDS) are common and severe pulmonary critical illness characterized by excessive pulmonary inflammation and dysfunction (1,2). The NOD-like receptor family, pyrin domain containing 3 (NLRP3) inflammasome is a multiprotein complex orchestrating innate immune response. In recent years, NLRP3 inflammasome and downstream caspase- 1 activation are shown to play a critical role in ALI caused by numbers of etiology including hemorrhagic shock, lipopolysaccharide (LPS) and mechanical ventilation (3-5). Therapies targeting caspase-1 shows great potential in critical diseases like sepsis (6). However, quite less is known about the causes of 
NLRP3 inflammasome activation in ALI, especially caused by non-infectious factors.

Activation of NLRP3 inflammasome requires both the initial inflammasome priming which serves to trigger increased transcriptions of inflammasome components and subsequent assembling of NLRP 3 , ASC and procaspase-1 (7). It has been shown that numbers of pathogen associated molecular patterns (PAMPs) were able to promote NLRP3 inflammasome activation $(8,9)$. Besides PAMPs, damage associated molecular patterns (DAMPs) including extracellular DNA were also able to facilitate NLRP3 inflammasome activation and promote organic inflammation and injury.

MtDNA, which contains high-frequency cytosinephosphate-guanine $(\mathrm{CpG})$ repeats, was one of the main components of mitochondrial DAMPs (10). The capacity of intracytoplasmic mtDNA to trigger innate immune responses through NLRP3 inflammasome was identified (11) and our previous study has also proven that mtDNA was able to induce pulmonary inflammation (12). However, the potential ability of extracellular mtDNA to induce NLRP3 inflammasome activation and its mechanism has not been fully understood. Different from intracytoplasmic mtDNA, extracellular mtDNA could not serve as a ligand of NLRP3 but was able to interact with TLR9 and induce the activation of MAPKs and NF- $\kappa B$ $(13,14)$. Our previous studies have indicated that serum mtDNA was significantly increased in polytrauma patients and extracellular mtDNA was able to induce aseptic pulmonary inflammation and IL- $1 \beta$ production via TLR9 $(12,15)$. In recent years, there has also been increasing interests in clarifying the role of endogenous danger signals in the development of inflammatory lung injury and ARDS/ ALI, especially in those caused by non-infectious causes $(14,16)$. Recently, it was shown that inhibition of TLR9 substantially diminished NLRP3 inflammasome activation and inflammatory pancreatic injury in acute pancreatitis mouse model (17). Although it has been demonstrated that TLR9 should be a key factor in the NLRP3 inflammasome activation in aseptic organic inflammation and injury, the molecular mechanism linking TLR9 and NLRP3 is still not clear and this study was designed to investigate the role of extracellular $\mathrm{mtDNA}$ in NLRP3 inflammasome activation in ALI.

\section{Methods}

Ethylene-bis (oxyethylenenitrilo) tetraacetic acid (EGTA), sucrose, 4-Morpholinepropanesulfonic acid (MOPS), phorbol 12-myristate 13-acetate (PMA) and protease inhibitor cocktail were purchased from Sigma. Belnacasan (VX-765) was obtained from Selleck. Pyrrolidinedithiocarbamic acid (PDTC) nuclear and cytoplasmic protein extraction kit, caspase 1 activity assay kit, colorimetric TUNEL apoptosis assay kit and Histone H3 antibody were obtained from Beyotime Co. Ltd. (Shanghai, China). Anti-procaspase-1 and anti-cleaved caspase-1 p20 antibody were purchased from Santa Cruz Biotechnology, Inc. Anti-NLRP3/NALP3 antibody was obtained from R\&D Systems. Antibodies to p38 MAPK and phospho-p38 MAPK (Thr180/Tyr182) were purchased from Cell Signaling Technology. Antibodies to GAPDH, Goat-anti-rabbit IgG-HRP, goat-anti-mouse IgG-HRP were obtained from Vazyme Biotech Co. Ltd. (Nanjing, China). Antibodies to NF-кB p65 subunit, ERK and phospho-ERK, anti- $\beta$-actin antibody was obtained from Abcam. Human IL-18/IL-1F4 ELISA kit was purchased from R\&D Systems and other ELISA kits were purchased from MultiSciences Biotech Co. Ltd (Hangzhou, China). RNA isolation kit was obtained from Tiandz (Beijing, China) and SYBR Green Master Mix was obtained from Vazyme Biotech (Nanjing, China). The small interfering RNA (siRNA) targeting caspase-1, NLRP3, p38 MAPK and TLR9 and negative control siRNA were purchased from GenePharma (Shanghai, China). Lipofectamine 2000 and Opti-MEM I reduced serum medium were purchased from Invitrogen Corporation (Carlsbad, CA, USA).

\section{Mice and ethics statement}

Healthy male C57BL/6 mice weighting 20-22 $\mathrm{g}$ and aging 7-8 weeks were purchased from Animal Feeding Center of Yangzhou University (Yangzhou, China). Animal cares were in accordance with the Institutional Animal Care and User guidelines. All the experimental processes were approved by the Model Animal Research Centre of Jingling Hospital.

\section{Cell line, culture, transfection and differentiation}

THP-1 human monocytes were obtained from American Type Culture Collection (ATCC). THP-1 cells were cultured in RPMI 1640-HEPES containing $100 \mathrm{U} / \mathrm{mL}$ penicillin/streptomycin and $10 \%$ fetal bovine serum and incubated at $37{ }^{\circ} \mathrm{C}$ in a $\mathrm{CO}_{2}$ incubator according to the supplier's guidelines. The siRNA transfection of THP1 cells was conducted by using Lipofectamine 2000 according to the manufacturer's recommended protocol 
(Invitrogen Corporation). Briefly, THP-1 cells were seeded at a density of $5 \times 10^{5}$ cells $/ \mathrm{mL}$ in 10 -cm dishes in $12 \mathrm{~mL}$ of non-antibiotic growth medium. The Lipofectamine 2000 and siRNA solution were separately prepared in $1.5 \mathrm{~mL}$ of Opti-MEM I reduced serum medium and were then mixed and incubated for 20 minutes at room temperature to generate the siRNA-Lipofectamine complex. Nextly, the mixed solution was added to THP-1 cells to a final siRNA concentration of $50 \mathrm{nM}$ and THP-1 cells were incubated in six-well plates at $37^{\circ} \mathrm{C}$. The knockdown efficiency was verified 36 to $72 \mathrm{~h}$ after transfection. For macrophage differentiation, THP- 1 cells were plated at $1 \times 10^{6}$ cells/ well in six-well plates by exposure to $100 \mathrm{ng} / \mathrm{mL}$ PMA for $24 \mathrm{~h}$ with no FBS. The differentiated, plastic-adherent cells were washed twice with RPMI 1640 and allowed to rest for 6 hours with fresh complete medium before exposure to various stimulators.

\section{$m t D N A$ preparation}

Mitochondrial DNA was extracted from the isolated mitochondria pellets of THP-1 cells and fresh mice liver in accordance with previous reported protocol by Christian Frezza (18). DNA purity, mtDNA purity and endotoxin pollution was detected as shown in our previous study (12). MtDNA extracted from THP-1 cells was used in the in vitro experiment while the mtDNA extracted from mice liver was applied to animal experiment.

\section{Animal procedures}

C57BL/6 mice were randomly divided into four groups with 12 animals per group. All of the mice were anesthetized by intraperitoneal pentobarbital sodium $(3 \mathrm{mg} / \mathrm{kg})$ and placed in supine with head tilted back. The intratracheal administration of PBS or mtDNA $(3 \mathrm{mg} / \mathrm{kg})$ in a volume of $60 \mu \mathrm{L}$ was performed by a microsprayer (Penn-Century, USA). For a subset of this experiment, mice were intraperitoneally pretreated with Belnacasan (VX-765) $(30 \mathrm{mg} / \mathrm{kg})$ or PBS for $2 \mathrm{~h}$ before mtDNA exposure. All of the mice were sacrificed $6 \mathrm{~h}$ after intratracheal administration. Lungs were harvested from six mice and bronchoalveolar lavage fluid (BALFs) were harvested from the other six mice in the same group. The larger left lobe was fixed in $4 \%$ paraformaldehyde for histological analysis, the middle right lobe was weight and dried to test Wet/Dry ratio and the remaining lung lobes were separately stored at $-80{ }^{\circ} \mathrm{C}$. Another 12 mice were untreated shams (Control) and were immediately sacrificed following tracheal intubation procedure and lungs and BALFs were also harvested as stated above. BALFs were obtained by the method as previous described (19).

\section{THP-1 macrophage stimulation process}

Differentiated THP-1 macrophages were exposed to mtDNA $(20 \mu \mathrm{g} / \mathrm{mL})$ or PBS. For the inhibition studies, THP-1 macrophages were pretreated with SB203580 (1 $\mu M$, p38 MAPK inhibitor) or PDTC $(10 \mu M$, $\mathrm{NF}-\kappa \mathrm{B}$ inhibitor), or were transfected with caspase-1 siRNA, NLRP3 siRNA, TLR9 siRNA or p38 MAPK siRNA before mtDNA exposure. The cell-conditioned medium was collected by centrifugation after exposure and then assayed for proinflammatory cytokines and caspase-1 activity according to manufacturer's protocols. THP-1 macrophages were lysed by extraction buffer containing protease inhibitor cocktail (Roche) and boiled with loading buffer for $30 \mathrm{~min}$. In parts of the study, to extract nucleoprotein, THP-1 macrophages were lysed and processed by using nuclear and cytoplasmic protein extraction kit according to the manufacturer's protocol.

\section{Histopathology of the lungs}

The larger left lobes were fixed overnight at $4{ }^{\circ} \mathrm{C}$ in $4 \%$ paraformaldehyde and processed by successive dehydration with an alcohol series and xylene. The tissues were then embedded in paraffin and cut into 5 - $\mu \mathrm{m}$-thick sections for hematoxylin-eosin (HE) staining or TdT-mediated dUTP Nick-End Labeling (TUNEL) apoptosis assay. HE staining was carried out according to the instructions provided by the manufacturer to determine the severity of the lung inflammation. The TUNEL apoptosis assay of lung was performed by Colorimetric TUNEL apoptosis assay kit according to the manufacture's protocol to determine the lung injury. All tissue cuts were evaluated by an experienced blinded pathologist and scored according to the criteria described previously (20).

\section{Quantitative reverse transcriptase-polymerase chain reaction ( $q R T-P C R)$}

RNA from THP-1 macrophages and mice lungs (upper lobe of right lung) were extracted by RNA isolation kit (Tiandz, Inc., Beijing China). qRT-PCR was performed by using ABI PRISM 7000 Sequence Detection System and 
SYBR Green Master Mix from Vazyme Biotech (Nanjing, China) according to manufacturer's protocol. Relative fold changes between different stimulations or pretreatments were calculated with the comparative Ct method ( $2^{-\Delta \Delta \mathrm{Ct}}$ Method).

\section{Supernatant and BALF concentration followed by caspase-1 activity assay}

Cell-conditioned supernatants and BALF were concentrated by Millipore UFC801024 centrifugal filter according to its protocol. Briefly, $3 \mathrm{~mL}$ supernatant or BALF was added into the upper part of the filter and then centrifuged at 4,000 $\mathrm{g}$ at $4{ }^{\circ} \mathrm{C}$ for $25 \mathrm{~min}$. After that, about $400 \mu \mathrm{L}$ concentrated liquid remained in the upper part and was aspirated out and analyzed immediately by caspase- 1 activity assay kit according to the protocol supplied by its manufacture.

\section{Western blot analysis}

Cell proteins were extracted from THP-1 macrophages and Western blot analysis was performed as previously described. Antibodies were diluted as follows: anti-betaactin antibody $(1: 1,000)$, anti-GAPDH antibody $(1: 2,000)$, anti-NLRP3 antibody (1:600), anti-cleavage caspase-1 p20 subunit antibody (1:500), anti-procaspase-1 antibody (1:1,000), anti-phospho-p38 MAPK (Thr180/Tyr182) antibody (1:800), anti-p38 MAPK antibody (1:800), antiERK antibody (1:800) and anti-phospho-ERK antibody (1:800) in PBST with 2\% BSA. The corresponding second antibodies were diluted at 1:10,000.

\section{Statistics}

Experimental results were expressed as the means \pm standard deviation (SD) and assessed for statistical significance by Student's $t$-test. Two-tailed $\mathrm{P}$ value $<0.05$ was considered as statistically significant. All statistical analyses were performed by using PASW Statistics 18.0 (IBM Corporation, Armonk, NY, USA).

\section{Results}

NLRP3 inflammasome activation and subsequent caspase-1 activation is critical in exogenous mtDNA-induced pulmonary inflammation and injury in mice

Previous reported studies has shown that intravenous injection of mtDNA was able to induce inflammation and injury of lung $(13,14)$ and another study conducted by our group has proven that intratracheal administration of mtDNA was also capable of trigging pulmonary inflammation in $6 \mathrm{~h}$ (12). Representative microscopic images and relevant quantitative examination and semiquantitative scores of lung injury are shown in Figure 1. MtDNA administration significantly induced excessive pulmonary inflammation and tissue damage in accordance with ALI. It is shown that mtDNA exposure caused abundant inflammatory cell infiltration, alveolar septa thickening and apoptosis of lung parenchymal cells. In addition, quantized data also indicated that mtDNA exposure would significantly increase the degree of lung injury and edema. All these results indicate that extracellular mtDNA is a critical mediator of ALI with increased lung inflammation permeability.

As IL- $1 \beta$ and IL-18 release and active caspase- 1 production has been shown to be hallmark of inflammasome activation. We therefore assayed IL-1 $\beta$, IL-18 and caspase-1 activity in BALF. It was suggested that mtDNA could significantly increase IL-1 $\beta / \mathrm{IL}-18$ production and caspase-1 activity in BALF (Figure $2 A, B, C$ ). MtDNA exposure also up-regulated the transcription of NLRP3 and ASC in mice lungs (Figure $2 D, E, F$ ). Further analysis of lung tissue also indicated that mtDNA was capable of inducing NLRP3 expression in lung and the production of activated p20 subunit of caspase-1 (Figure 2G,H,I). All these results demonstrated that local instillation of mtDNA could induce NLRP3 inflammasome priming and activation in mice lungs.

To further confirm the role of NLRP3 inflammasome in mtDNA-induced lung inflammation and injury, we conducted inhibition experiment in mice. As no NLRP3 inhibitor is available for selling then, we adopted belnacasan (VX-765), a selective caspse-1 inhibitor, to verify the role of inflammasome in mtDNA-induced lung inflammation and injury. It was revealed that belnacasan significantly attuned mtDNA-induced inflammation and injury (Figure 3). Additionally, belnacasan dramatically decreased mtDNAinduced IL-1 $\beta$ and IL-1 8 production. These results indicated that NLRP3 inflammasome and downstream caspase-1 played a key role in mtDNA-induced lung inflammation and injury.

\section{mtDNA exposure leads to NLRP3 inflammasome priming and activation in PMA-primed THP-1 macrophages}

Given that macrophage is a primary subset of inflammation- 
A

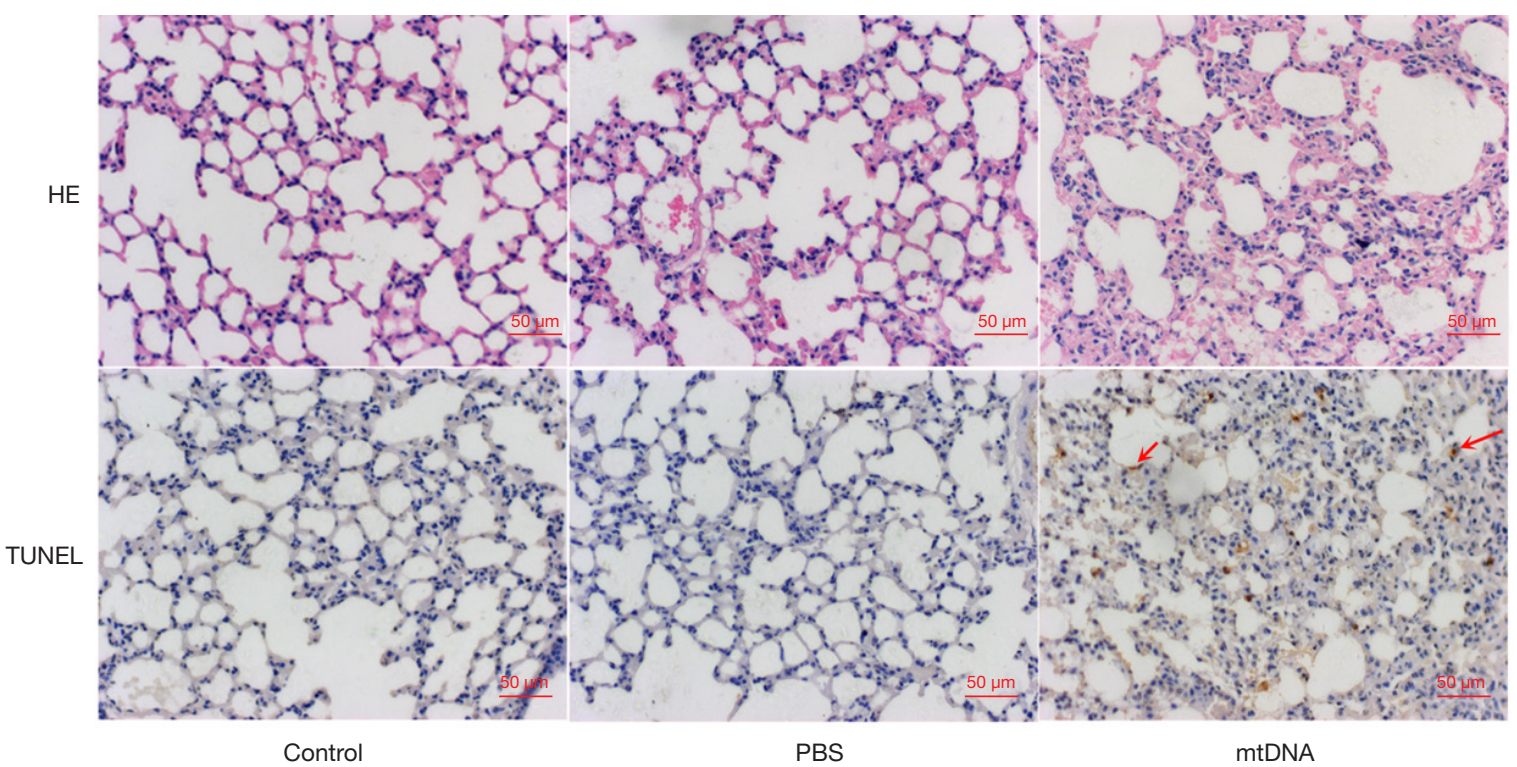

B
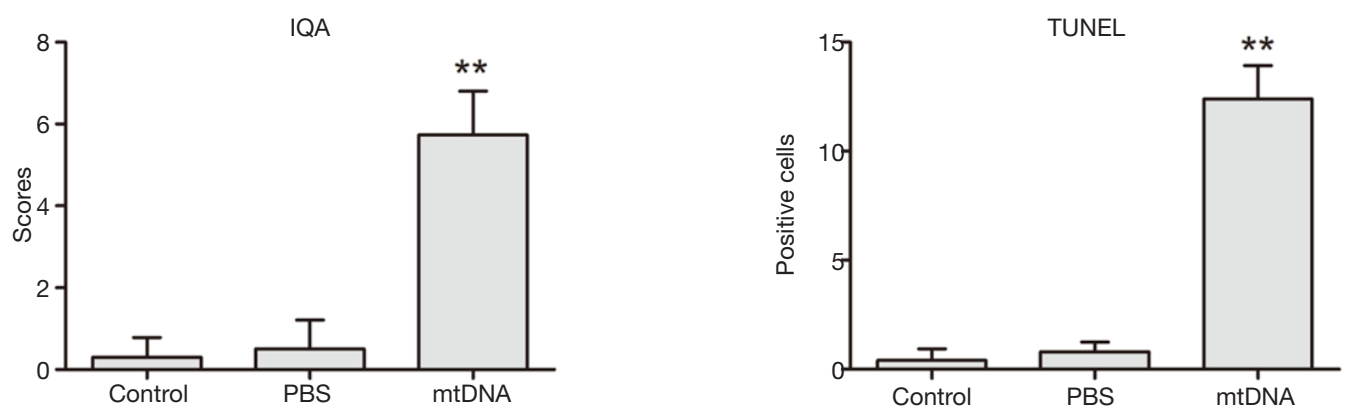

Cells in BALF
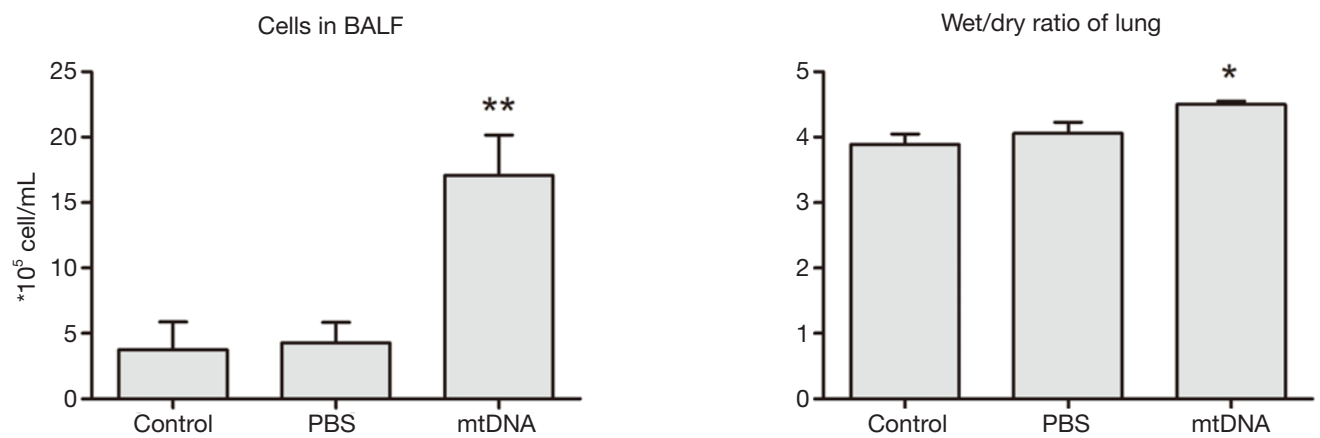

Figure 1 Intratracheal mtDNA administration causes pulmonary inflammation and injury. (A) Intratracheal mtDNA administration for $6 \mathrm{~h}$ significantly induces lung inflammation; (B) histological score of lung injury (IQA), lung cell apoptosis, cells in bronchoalveolar lavage fluid (BALFs) and lung wet/dry ratio are significantly increased in mtDNA exposure group. Arrow: TUNEL positive cells; ${ }^{*} \mathrm{P}<0.05 ;{ }^{* *} \mathrm{P}<0.01$.

regulating cells in lung and plays a crucial role in mtDNAinduced lung inflammation (21), we adopted PMA-primed THP-1 macrophages. Differentiated THP-1 macrophages were incubated with either PBS or mtDNA $(20 \mu \mathrm{g} / \mathrm{mL})$. MtDNA exposure is capable of inducing caspase- 1 activation and IL-1 $\beta$, IL-18 and TNF- $\alpha$ release (Figure $4 A, B, C$ ). It was also demonstrated that caspase- 1 p20 subunit was substantially increased in mtDNA stimulated concentrated supernatant (Figure 4D). Nextly, we synthesized caspase-1 siRNA to verify its role in mtDNA-induced inflammation. 
A

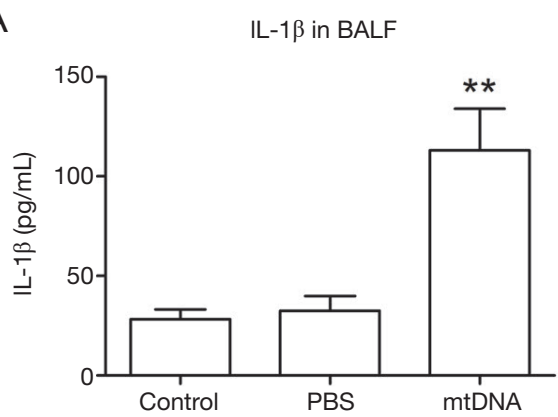

D

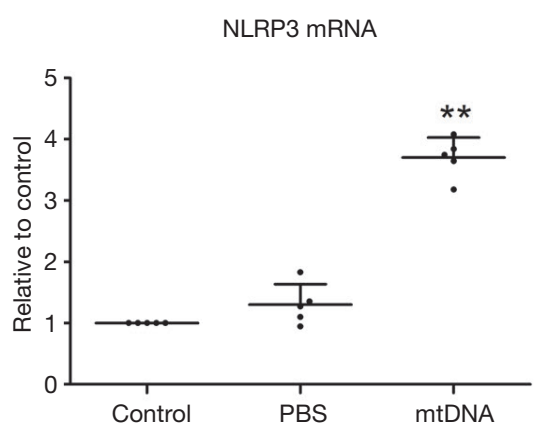

G

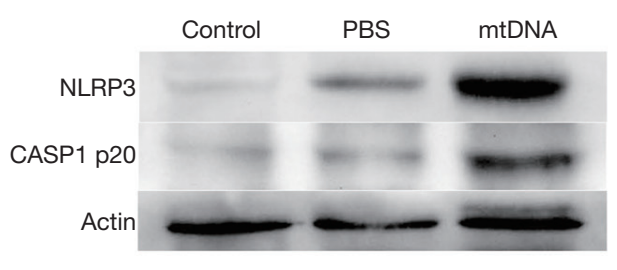

B

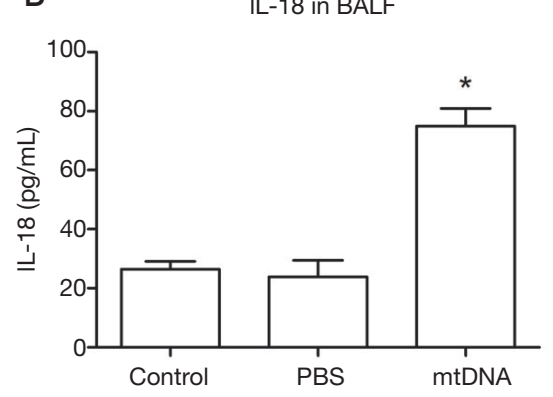

$\mathrm{E}$

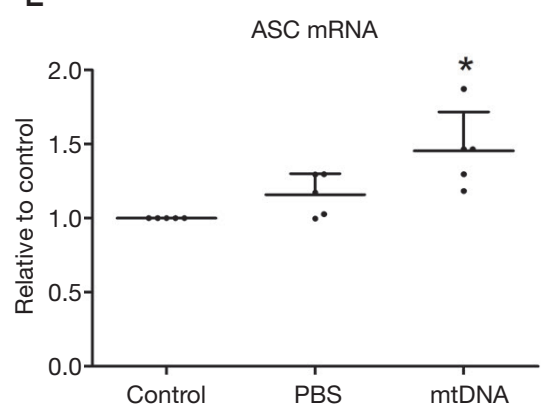

H NLRP3

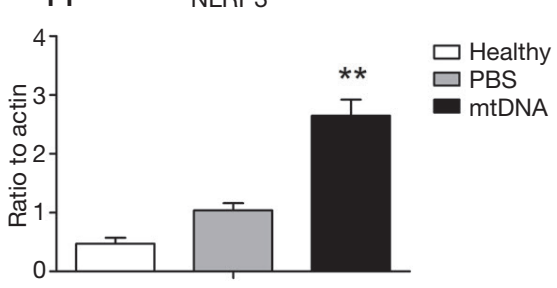

C

Caspase-1 activity in BALF

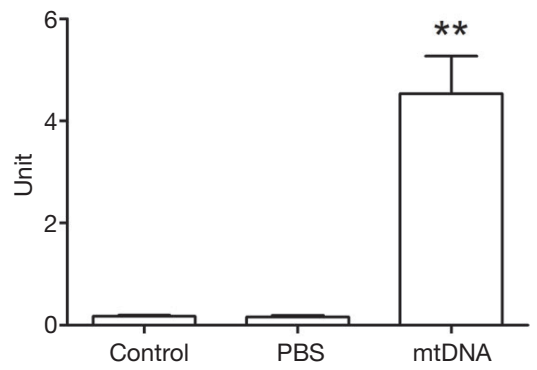

$\mathrm{F}$

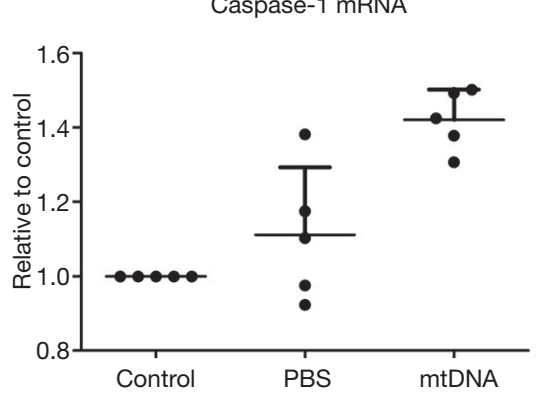

I Caspase-1 p20

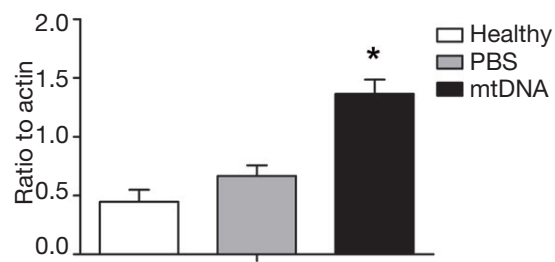

Figure 2 Intratracheal mtDNA administration induces NLRP3 inflammasome activation in lung. (A,B,C) mtDNA exposure significantly increased IL-1 $\beta$, IL-18 release and caspase-1 activation; (D,E,F) mtDNA exposure significantly increased NLRP3 and ASC transcription in lung; $(\mathrm{G}, \mathrm{H}, \mathrm{I}) \mathrm{mtDNA}$ exposure induces NLRP3 expression and caspase-1 $\mathrm{p} 20$ subunit production. ${ }^{*} \mathrm{P}<0.05,{ }^{* *} \mathrm{P}<0.01$.

As shown in Figure 4E, transfection of caspase-1 siRNA of $50 \mathrm{nM}$ for $60 \mathrm{~h}$ could significantly decrease caspase-1 transcription. Then transfected THP-1 macrophages were stimulated with mtDNA followed by ELISA analysis of IL-1 $\beta$, IL-18 and TNF- $\alpha$ from culture. The results showed that inhibition of caspase- 1 or transfection of caspase- 1 siRNA led to significant reduction of IL- $1 \beta$ and IL-18 while no effect on TNF- $\alpha$ were detected (Figure $4 F$ ). These results revealed that mtDNA-induced caspase-1 activation was a key pro-inflammatory process.

As NLRP3 priming has been shown to be a critical step and mtDNA is a typical DAMPs, we next assayed transcription of NLRP3 inflammasome components after mtDNA stimulation. It was shown that mtDNA exposure could induce early increased transcription of
NLRP3, ASC and procaspase-1 (Figure 5A). In contrast to NLRP3 inflammasome components, extracellular mtDNA stimulation is able to induce persistent increased NF- $\kappa \mathrm{B}$ transcription. We also detected NLRP3 and procaspase-1 in mtDNA-stimulated THP-1 macrophage and it was indicated that mtDNA could induce increased NLRP3 and procaspase- 1 expression and a mild decrease of intracellular procaspase- 1 at $6 \mathrm{~h}$ (Figure $5 B$ ). We then synthesized NLRP3 siRNA to check its roles. Silencing of NLRP3 significantly decreased mtDNA-induced IL- $1 \beta$ production while no effect on TNF- $\alpha$ production was observed (Figure 5C). These results indicated that extracellular mtDNA stimulation was capable of inducing NLRP3 inflammasome priming and activation followed by IL-1 family cytokine production. 
A

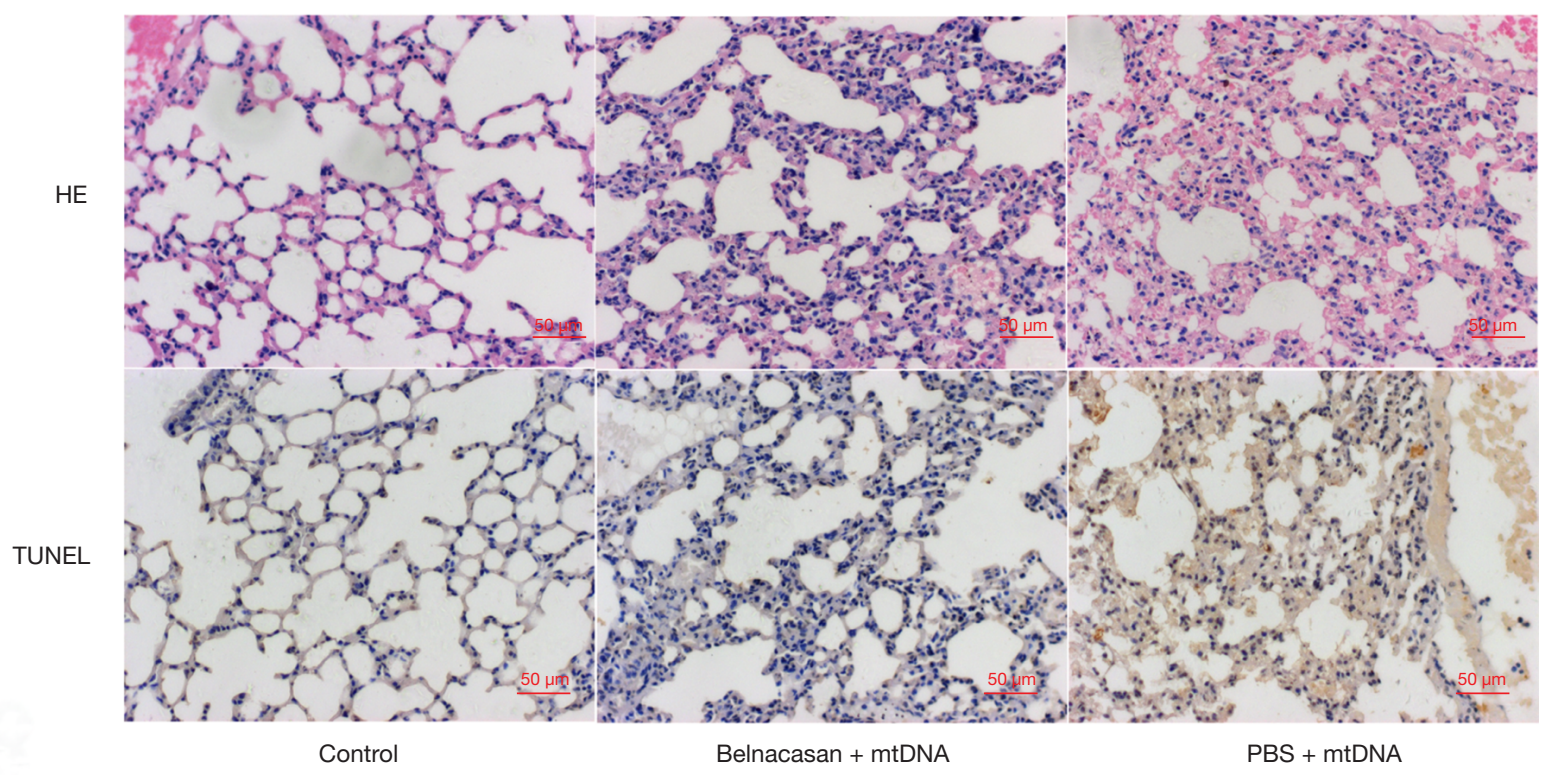

B
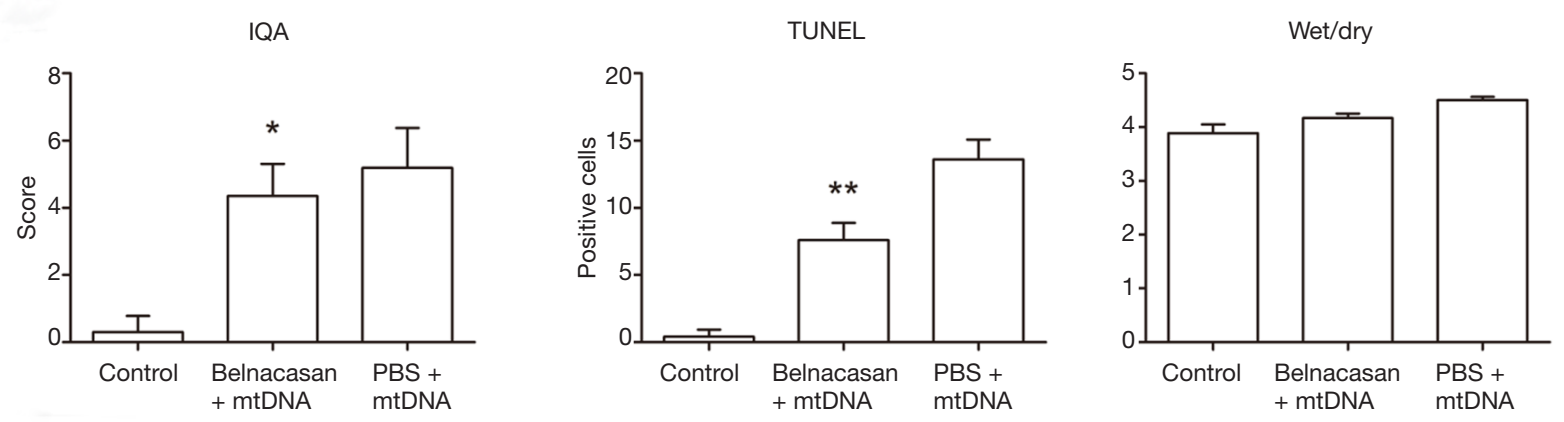

C
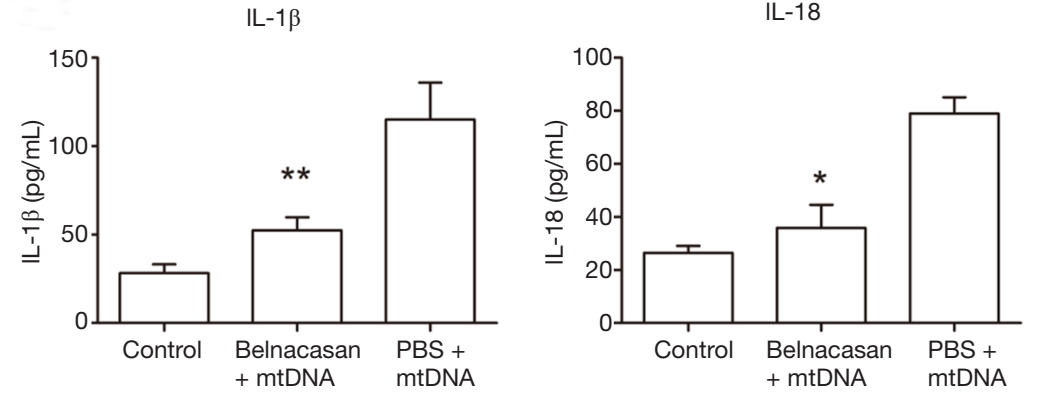

Figure 3 Caspase-1 is essential in mtDNA-induced lung inflammation and injury. (A) Belnacasan significantly decreased mtDNA-induced pulmonary inflammation; (B) histological score of lung injury (IQA), lung cell apoptosis and lung wet/dry ratio is significantly decreased in belnacasan treated group; (C) IL- $1 \beta$ and IL-18 is significantly decreased in belnacasan treated group. ${ }^{*} \mathrm{P}<0.05,{ }^{* *} \mathrm{P}<0.01$.

\section{TLR9, p38 MAPK and NF- $\kappa B$ participate in mtDNA- induced NLRP3 inflammasome activation}

Given that TLR9 has been accepted as a double-stranded DNA receptor, we examined whether TLR9, p38 MAPK and NF- $\mathrm{BB}$ also participate in mtDNA-induced NLRP3 inflammasome priming and activation. As shown in Figure 6, silencing of TLR9 significantly decreased mtDNA-induced IL- $1 \beta$ and TNF- $\alpha$ production and NLRP3 expression. Since both ERK and p38 MAPK have been reported to participant in mtDNA-induced inflammation in neutrophils (13), we also examined their roles in mtDNA-induced NLRP3 


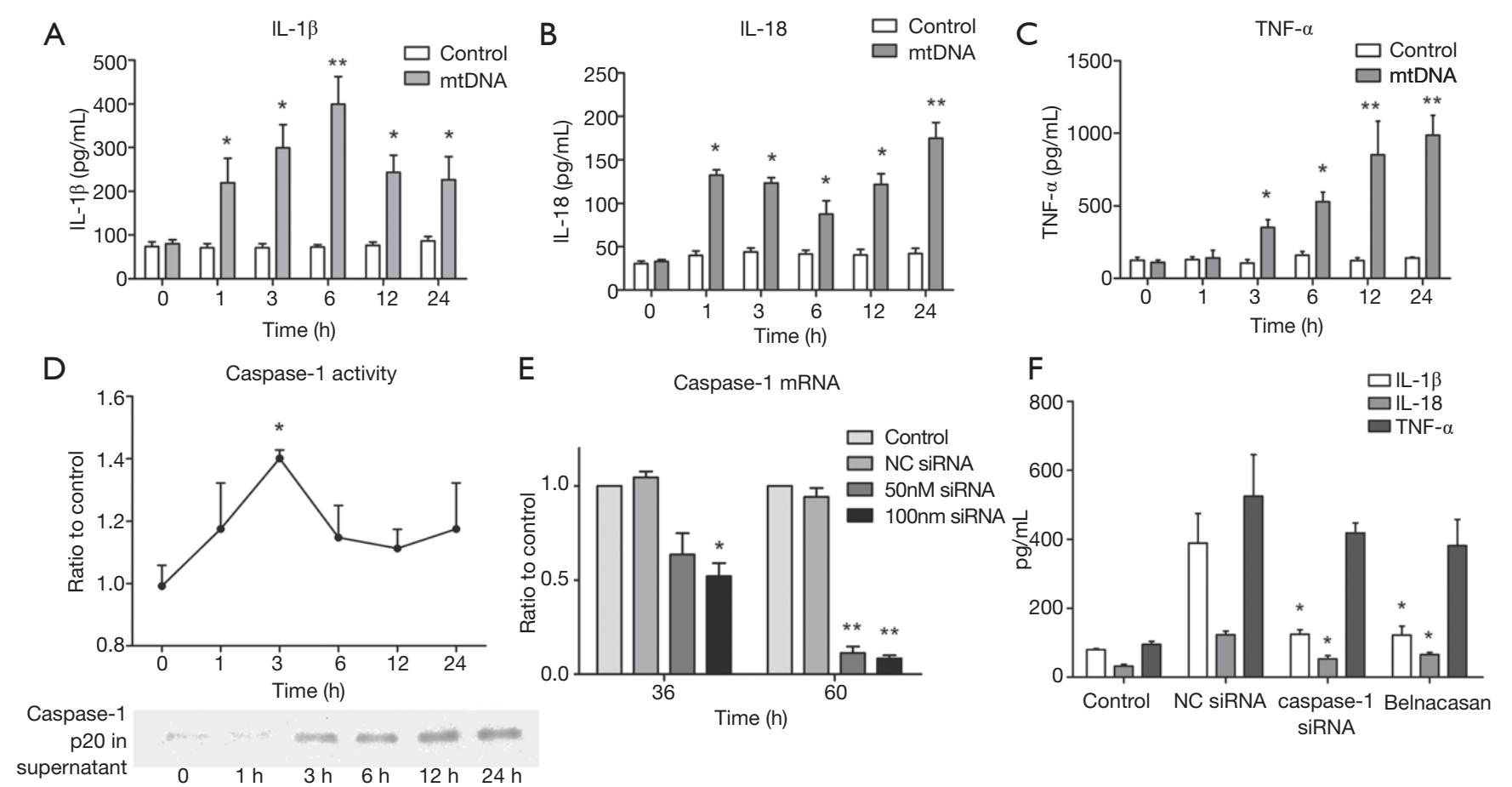

Figure 4 Extracellular mtDNA promotes IL-1 family cytokine production and caspase-1 activation in THP-1 macrophages. (A,B,C) Extracellular mtDNA promotes IL-1 $\beta, \mathrm{IL}-18$ and TNF- $\alpha$ production; (D) extracellular mtDNA promotes caspase- 1 activation and release; (E) knock-down efficiency of caspase-1 siRNA; (F) inhibition or knock-down of caspase-1 significantly decreases mtDNA-induced IL-1 family cytokine production but has no effect on TNF- $\alpha .{ }^{*} \mathrm{P}<0.05,{ }^{* *} \mathrm{P}<0.01$.

inflammation priming in THP-1 macrophages. As shown in Figure $7 A$, mtDNA exposure induced a dramatic increase of phosphorylated p38 MAPK (p-p38 MAPK) while no significant changes of phosphorylated ERK were detected. MtDNA exposure could induce increased nuclear translocation of NF- $\mathrm{KB}$ (Figure $7 A$ ). To further examine $\mathrm{p} 38$ MAPK and NF- $\mathrm{KB}$ roles in extracellular mtDNA-induced NLPR3 inflammasome priming, we adopted p38 MAPK siRNA, SB203580 (a selective inhibitor of p38 MAPK) and PDTC (a selective inhibitor of NF- $\mathrm{kB}$ p65 nuclear translocation). It was shown that silencing of p38 MAPK and inhibition of $\mathrm{p} 38 \mathrm{MAPK}$ or NF- $\mathrm{KB}$ could suppress mtDNA-induced IL-1 $\beta$ production (Figure $7 B$ ) and NLRP3 expression in THP-1 macrophage (Figure 7C,D). Interestingly, we also found that mtDNA related NF- $\mathrm{KB}$ p65 nuclear translocation was only partially mediated by p38 MAPK (Figure 7C) which indicated that p38 MAPK is a dominate but not the only mediator in this process. All these results indicated that extracellular mtDNA was capable of inducing TLR9-p38 MAPK pathway activation and subsequent NF- $\kappa$ B p65 nuclear translocation, which promoted NLRP3 inflammasome priming and activation.

\section{Discussion}

This present study indicated that extracellular mtDNA in lung could induce NLRP3 inflammation activation which resulted in pulmonary inflammation and injury. Further in vitro experiments demonstrated that the interaction of mtDNA and TLR9 followed by $\mathrm{p} 38$ MAPK phosphorylation and NF- $\kappa B$ activation are crucial in extracellular mtDNAinduced NLRP3 inflammation priming and activation. These findings revealed that extracellular mtDNA was also able to induce NLRP3 inflammation activation in addition to cytoplasmic oxidized mtDNA. To the best of our knowledge, this study provides the first evidence that extracellular mtDNA in lung is a source of NLRP3 inflammation priming and activation which promotes excessive pulmonary inflammation and injury.

Consistent with our previous study, it was shown that intratracheal administration of mtDNA for $6 \mathrm{~h}$ could induce pulmonary inflammation response which is characterized by increased inflammatory cell infiltrating, alveolar septa thickening, vascular congestion and pro-inflammatory cytokines (IL-1 $\beta$ and IL-18) production. Beyond that, we 
A

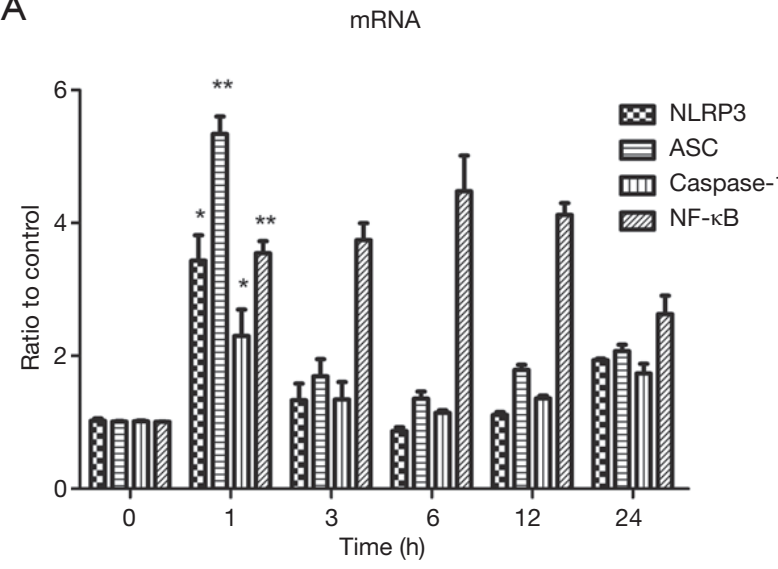

B

NLRP3

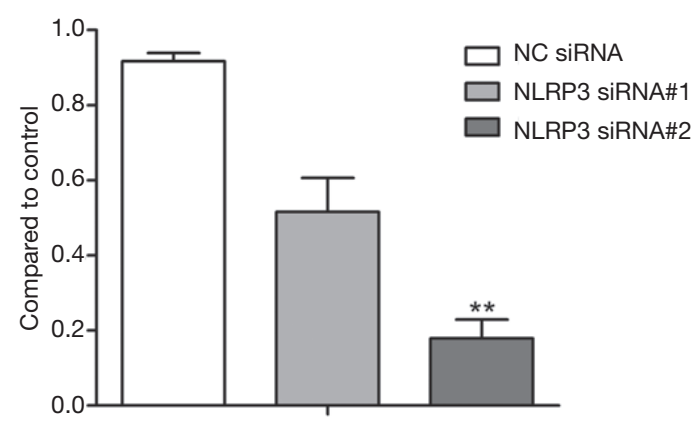

C

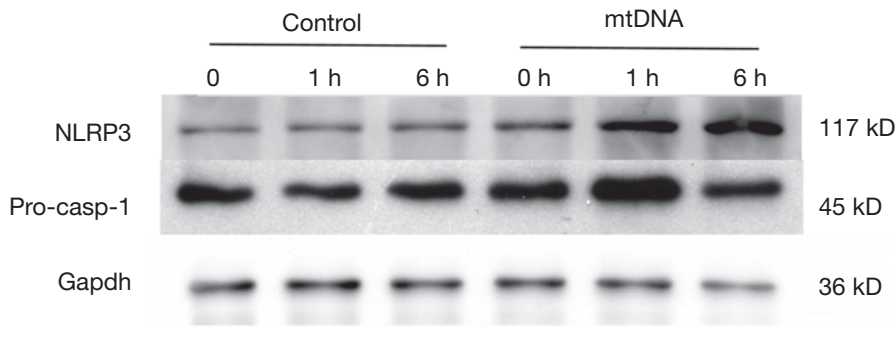

$\mathrm{D}$

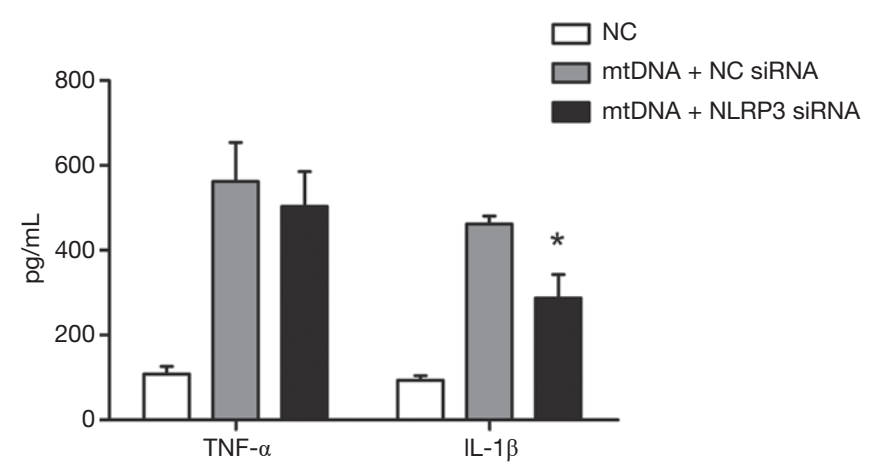

Figure 5 Extracellular mtDNA promotes NLRP3 inflammasome priming and activation in THP-1 macrophages. (A) Extracellular mtDNA exposure significantly increased NLRP3, ASC, caspase-1 and NF- $-\mathrm{B}$ transcription; (B) knock-down efficiency of NLRP3 siRNA; (C) extracellular mtDNA exposure significantly increased NLRP3 and procaspase-1 expression; (D) knock-down of NLRP3 significantly decreases mtDNA-induced IL-1 $\beta$ production but has no effect on TNF- $\alpha .{ }^{*} \mathrm{P}<0.05,{ }^{* *} \mathrm{P}<0.01$.

also detected cell apoptosis in mtDNA-stimulated lung and it was indicated that intratracheal mtDNA administration could promote cell apoptosis in lung tissue. Considering that mtDNA exposure also caused increased lung permeability, these results demonstrated that extracellular mtDNA should be one of the sources of lung inflammation and pulmonary alveolar capillary barrier damage.

Although previous studies have demonstrated that NLRP3 inflammasome play a critical role in inflammatory lung diseases including ALI $(3,4,22,23)$, much less is known about the cause of it. Our study firstly indicated that extracellular mtDNA should be one of the sources of NLRP3 inflammation activation which resulted in pulmonary inflammation and injury. We demonstrated that mtDNA exposure substantially increased the release of IL-1 family cytokines and cleavaged caspase- 1 . This indicated that extracellular mtDNA stimulation could induce the hydrolysis of procaspase- 1 which is a key process of inflammasome activation. We also found that extracellular mtDNA significantly increased the content of NLRP 3 and caspase-1 p20 subunit in lung. Furthermore, in vivo experiments indicated that inhibition of caspase-1, the main effector of NLRP3 inflammasome activation, as expected decreased mtDNA induced lung injury and inflammation. These results demonstrated that extracellular mtDNA was an inducer of NLRP3 inflammasome in lung, which promoted pulmonary inflammation and injury. Since increased transcription of NLRP3 inflammasome components is the basis of substantial assembling and activation, we also assayed the transcription of these molecules. The transcription of NLRP3 and ASC was significantly increased in mtDNA instilled mice while no significant difference of caspase-1 transcription was detected. 

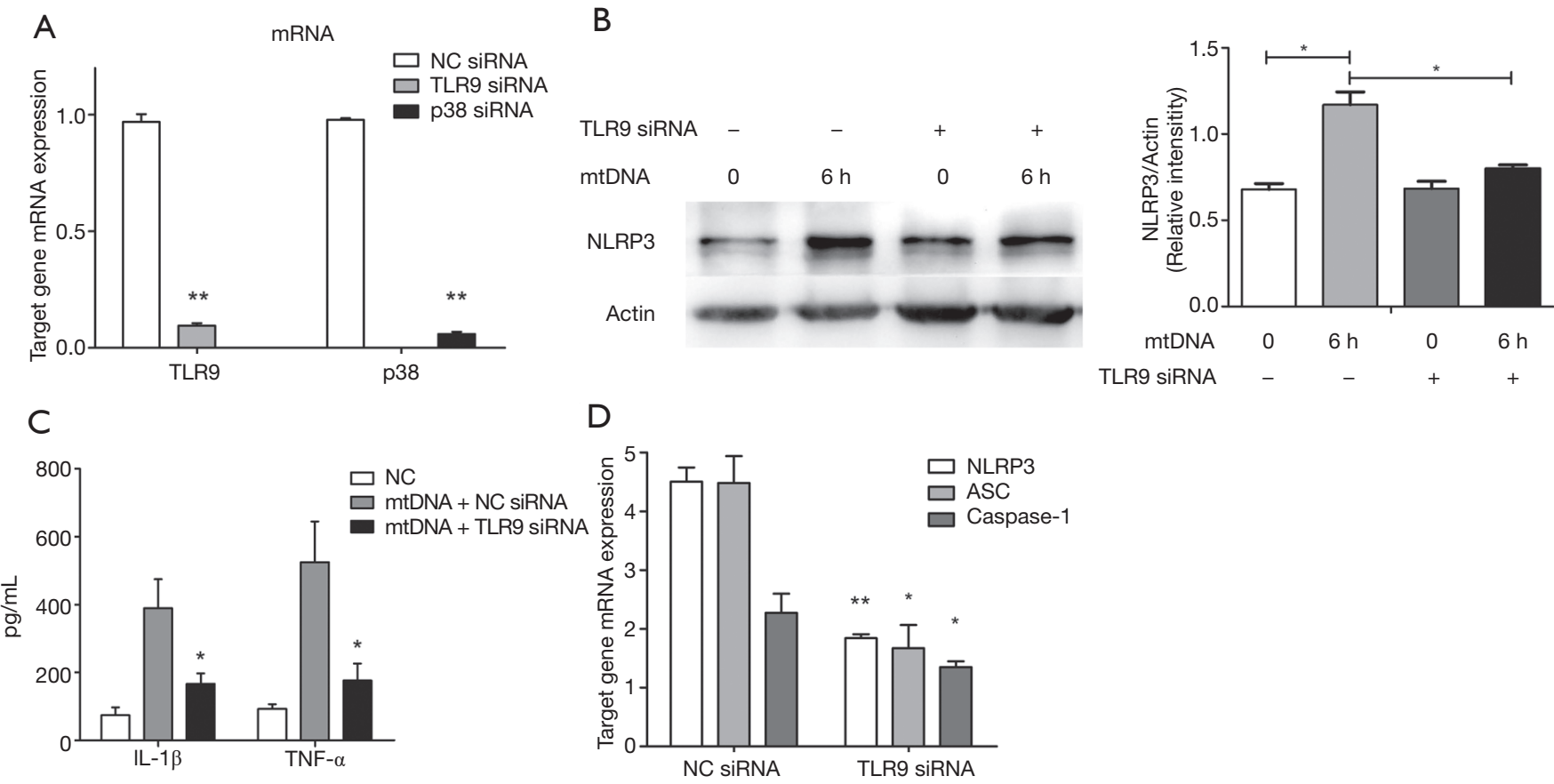

Figure 6 TLR9 is essential in extracellular mtDNA-induced NLRP3 inflammasome priming and activation. (A) Knock-down efficiency of TLR9 siRNA and p38 MAPK siRNA; (B) knock-down of TLR9 significantly neutralized extracellular mtDNA-induced NLRP3 upregulation; (C) knock-down of TLR9 significantly decreased mtDNA-induced IL-1 $\beta$ and TNF- $\alpha$ production; (D) knock-down of TLR9 significantly decreased extracellular mtDNA-induced NLRP3 inflammasome transcription. ${ }^{*} \mathrm{P}<0.05$.

Taking together, these results indicated that extracellular mtDNA in lung was able to induce increased transcription and expression of NLRP3 inflammasome components (priming) and promote NLRP3 inflammasome activation and pulmonary inflammation.

TLR9 has long been known to be a receptor for doublestrand DNA including mtDNA (24). However, its role in extracellular mtDNA-induced NLRP3 inflammasome activation is rarely known. In accordance with the in vivo experiments, the in vitro study indicated that extracellular mtDNA was able to induce NLRP3 inflammasome priming and activation through TLR9 in THP-1 macrophages. It was shown that mtDNA exposure rapidly increased the expression of NLRP3, procaspase-1 cleavage and IL-1 family cytokine production. These indicated that extracellular mtDNA promoted NLRP3 inflammasome priming and activation. Although several studies have proven that TLR9 should be a key upstream molecule in NLRP3 inflammasome activation $(17,25,26)$, there is still little evidence that could explain the mechanism between them, especially in the case of mtDNA stimulation. Thus, we explored the role of TLR9 and its downstream molecules in mtDNA-induced
NLRP3 inflammation priming and activation. Different from previous studies demonstrating that mtDNA could induce both p38 MAPK and ERK activation in neutrophils (13), our study indicated that extracellular mtDNA was capable of inducing phosphorylation of p38 MAPK but not ERK. Further investigation also indicated that inhibition of $\mathrm{p} 38$ MAPK pathway by p38 siRNA or SB203580 significantly diminished mtDNA-induced NLRP3 inflammasome priming and activation.

Although previous study has shown that mtDNA could increase phosphorylated NF- $\kappa$ B p65 in macrophages (14), $\mathrm{NF}-\kappa \mathrm{B}$ p65 in nucleus has not previously been reported and no previous study have explored the connecting molecular mediating the signaling between TLR9 and NF- $\kappa$ B. Moreover, no evidence of the role of NF- $\mathrm{KB}$ in extracellular mtDNA-induced inflammation and NLRP3 inflammasome priming has been reported. As $\mathrm{p} 38$ MAPK has been shown to be a key downstream molecule of TLR9, we detected the role of NF- $\mathrm{\kappa B}$ and its relationship with p38 MAPK in mtDNA-induced NLRP3 inflammasome activation. It was shown that mtDNA stimulation significantly increased $\mathrm{NF}-\kappa \mathrm{B}$ p 65 subunit nuclear translocation and inhibition of 
A

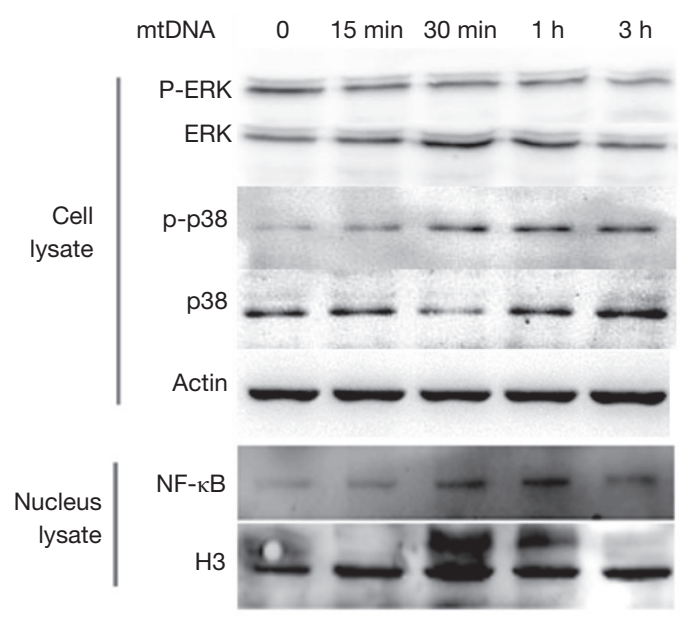

C

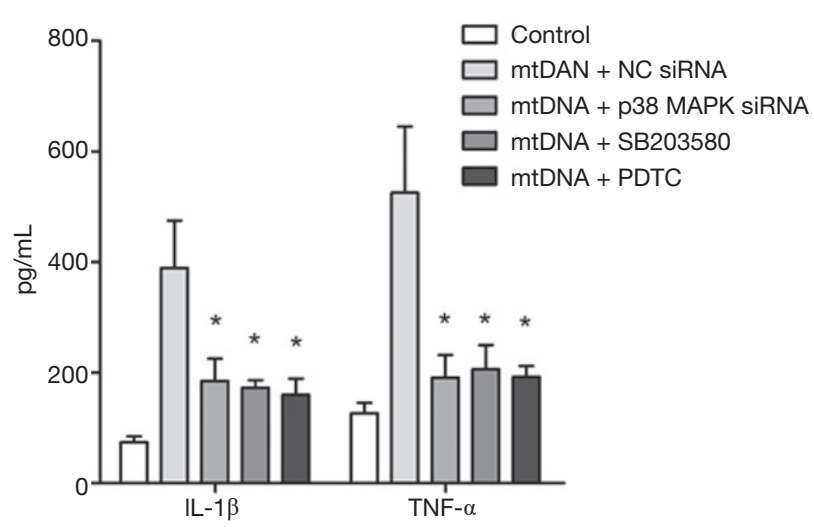

B

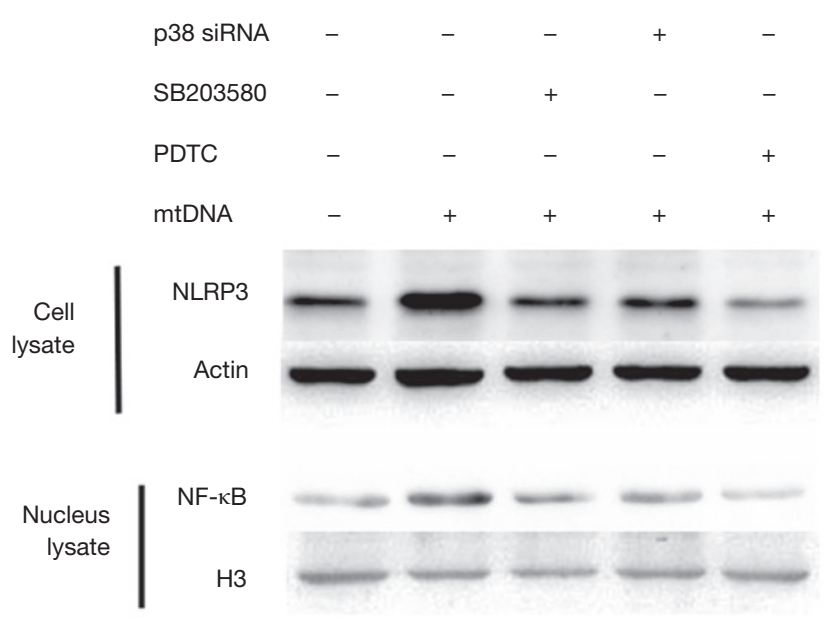

D

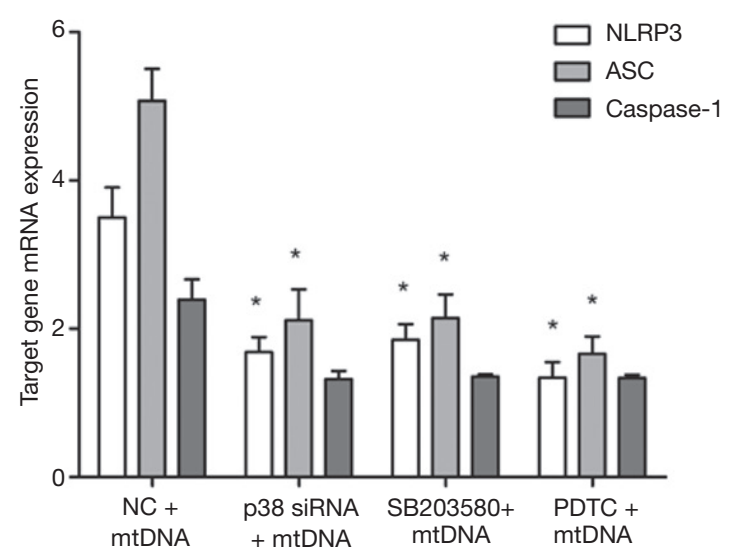

Figure 7 Extracellular mitochondrial DNA (mtDNA) promotes NLRP3 inflammasome priming through p38 MAPK and NF-kB. (A) Extracellular mtDNA promotes p38 MAPK and NF- $\mathrm{kB}$ translocation in THP-1 macrophages; (B) inhibition of p38 MAPK or NF- $\mathrm{kB}$ significantly inhibited mtDNA-induced NLRP3 up-regulation; (C) inhibition of p38 MAPK or NF-kB significantly inhibited mtDNAinduced IL-1 and TNF- $\alpha$ production; (D) inhibition of p 38 MAPK or NF- $\mathrm{kB}$ significantly decreased extracellular mtDNA-induced NLRP3 inflammasome transcription. ${ }^{*} \mathrm{P}<0.05$.

NF- $\kappa \mathrm{B}$ substantially decreased mtDNA-induced NLRP3 transcription and expression and caspase-1 cleavage which indicated NLRP3 inflammasome activation. Interestingly, it was also indicated that mtDNA-induced nuclear translocation of NF- $\kappa$ B p65 subunit was only partially mediated by 38 MAPK and other kinases or molecules like TAK1 (TGF-activated kinase) might also partially participate in this process. These results demonstrated that extracellular mtDNA induce NLRP3 inflammasome priming and activation through TLR9- 38 MAPK- NF-кB pathway.

Despite that cytoplasmic oxidized mtDNA has been reported to be able to induce NLRP3 inflammasome activation by binding with NLRP3 $(11,27)$, the role of extracellular mtDNA in NLRP3 inflammasome activation has not been revealed. Furthermore, extracellular DAMPs have always been treated as key factors in the initiation and amplification of inflammation. Our study demonstrated that extracellular mtDNA was able to induce NLRP3 inflammasome priming and activation by ways different from cytoplasmic oxidized mtDNA. As mtDNA has been shown to be released in a subset of histopathological conditions, this might be able to partially explain the NLRP3 inflammasome 
activation in ALI and other inflammatory lung diseases by non-infectious etiology. Our future research is partially focusing on verify the proportion of extracellular nonoxidized mtDNA under different pathological states. More recently, it was reported that NLRP3 inflammasome could also induce self-amplification and activation of nearby cells by release of incomplete NLRP3 inflammasome macromolecular complexes and ASC speckles $(28,29)$. This also gave an index to the importance of extracellular DAMPs in inflammation amplification. Additionally, targeting caspase- 1 has been considered to be a new approach in sepsis treatment (6) and a small-molecule inhibitor of the NLRP3 inflammasome with outstanding treatment potentials in inflammatory diseases has also been developed (30). NLRP3 inflammasome and its downstream caspase-1 showed exciting potential therapeutic targets for inflammatory diseases. Further detection of mtDNA, NLRP3, caspase-1 and IL-1 family in ARDS patients should be able to distinguish highrisk patients.

Although our study demonstrated that extracellular mtDNA could induce NLRP3 inflammasome priming and activation through TLR9- p38 MAPK- NF- $\mathrm{BB}$ in THP-1 macrophages, it was also shown that p38 MAPK should not be the exclusive molecular in the activation of $\mathrm{NF}-\kappa \mathrm{B}$ caused by extracellular mtDNA. Despite this, we illustrated the effect of extracellular mtDNA on NLRP3 inflammasome priming and activation in lung for the first time and provided evidences for potential NLRP3 inhibitors as therapeutics in ALI/ARDS.

In conclusion, this study demonstrates that extracellular mtDNA could promote NLRP3 inflammasome activation, acute inflammation and tissue damage in lung through TLR9, p38 MAPK and NF-кB pathway.

\section{Acknowledgments}

All authors are deeply grateful to Professor Xiaodong Han of Nanjing University for her kind help.

Funding: This work was supported by the National Natural Science Foundation of China (grant number 81370172 to Yong Song, 81570078 to Yong Song) and National Science and Technology Major Project (grant number 2014ZX09J14107-09C to Yong Song).

\section{Footnote}

Conflicts of Interest: The authors have no conflicts of interest to declare.
Ethical Statement: The authors are accountable for all aspects of the work in ensuring that questions related to the accuracy or integrity of any part of the work are appropriately investigated and resolved. Animal cares were in accordance with the Institutional Animal Care and User guidelines. All the experimental processes were approved by the Model Animal Research Centre of Jingling Hospital.

\section{References}

1. Zambon M, Vincent JL. Mortality rates for patients with acute lung injury/ARDS have decreased over time. Chest 2008;133:1120-7.

2. Matthay MA, Ware LB, Zimmerman GA. The acute respiratory distress syndrome. J Clin Invest 2012;122:2731-40.

3. Dolinay T, Kim YS, Howrylak J, et al. Inflammasomeregulated cytokines are critical mediators of acute lung injury. Am J Respir Crit Care Med 2012;185:1225-34.

4. Jones HD, Crother TR, Gonzalez-Villalobos RA, et al. The NLRP3 inflammasome is required for the development of hypoxemia in LPS/mechanical ventilation acute lung injury. Am J Respir Cell Mol Biol 2014;50:270-80.

5. Xiang M, Shi X, Li Y, et al. Hemorrhagic shock activation of NLRP3 inflammasome in lung endothelial cells. J Immunol 2011;187:4809-17.

6. Matute-Bello G. Targeting caspase-1 in sepsis: a novel approach to an old problem. Intensive Care Med 2007;33:755-7.

7. Sutterwala FS, Haasken S, Cassel SL. Mechanism of NLRP3 inflammasome activation. Ann N Y Acad Sci 2014;1319:82-95.

8. Kalantari P, DeOliveira RB, Chan J, et al. Dual engagement of the NLRP3 and AIM2 inflammasomes by plasmodium-derived hemozoin and DNA during malaria. Cell Rep 2014;6:196-210.

9. Bauernfeind F, Bartok E, Rieger A, et al. Cutting edge: reactive oxygen species inhibitors block priming, but not activation, of the NLRP3 inflammasome. J Immunol 2011;187:613-7.

10. Krysko DV, Agostinis P, Krysko O, et al. Emerging role of damage-associated molecular patterns derived from mitochondria in inflammation. Trends Immunol 2011;32:157-64.

11. Shimada K, Crother TR, Karlin J, et al. Oxidized mitochondrial DNA activates the NLRP3 inflammasome during apoptosis. Immunity 2012;36:401-14. 
12. Gu X, Wu G, Yao Y, et al. Intratracheal administration of mitochondrial DNA directly provokes lung inflammation through the TLR9-p38 MAPK pathway. Free Radical Biology and Medicine 2015;83:149-58.

13. Zhang Q, Raoof M, Chen Y, et al. Circulating mitochondrial DAMPs cause inflammatory responses to injury. Nature 2010;464:104-7.

14. Zhang JZ, Liu Z, Liu J, et al. Mitochondrial DNA induces inflammation and increases TLR9/NF-kappaB expression in lung tissue. Int J Mol Med 2014;33:817-24.

15. Gu X, Yao Y, Wu G, et al. The plasma mitochondrial DNA is an independent predictor for post-traumatic systemic inflammatory response syndrome. PLoS One 2013;8:e72834.

16. Sun S, Sursal T, Adibnia Y, et al. Mitochondrial DAMPs increase endothelial permeability through neutrophil dependent and independent pathways. PLoS One 2013;8:e59989.

17. Hoque R, Sohail M, Malik A, et al. TLR9 and the NLRP3 inflammasome link acinar cell death with inflammation in acute pancreatitis. Gastroenterology 2011;141:358-69.

18. Frezza C, Cipolat S, Scorrano L. Organelle isolation: functional mitochondria from mouse liver, muscle and cultured fibroblasts. Nat Protoc 2007;2:287-95.

19. Bhatnagar S, Shinagawa K, Castellino FJ, et al. Exosomes released from macrophages infected with intracellular pathogens stimulate a proinflammatory response in vitro and in vivo. Blood 2007;110:3234-44.

20. Sun Z, Wang C, Shi C, et al. Activated Wnt signaling induces myofibroblast differentiation of mesenchymal stem cells, contributing to pulmonary fibrosis. Int J Mol Med 2014;33:1097-109.

Cite this article as: $\mathrm{Wu} \mathrm{G}$, Zhu Q, Zeng J, Gu X, Miao Y, Xu W, Lv T, Song Y. Extracellular mitochondrial DNA promote NLRP3 inflammasome activation and induce acute lung injury through TLR9 and NF-кB. J Thorac Dis 2019;11(11):4816-4828. doi: $10.21037 /$ jtd.2019.10.26
21. Byrne AJ, Mathie SA, Gregory LG, et al. Pulmonary macrophages: key players in the innate defence of the airways. Thorax 2015;70:1189-96.

22. Fukumoto J, Fukumoto I, Parthasarathy PT, et al. NLRP3 deletion protects from hyperoxia-induced acute lung injury. Am J Physiol Cell Physiol 2013;305:C182-9.

23. Grailer JJ, Canning BA, Kalbitz M, et al. Critical role for the NLRP3 inflammasome during acute lung injury. J Immunol 2014;192:5974-83.

24. West AP, Koblansky AA, Ghosh S. Recognition and signaling by toll-like receptors. Annu Rev Cell Dev Biol 2006;22:409-37.

25. Neumann K, Ruland J. Kinases conquer the inflammasomes. Nat Immunol 2013;14:1207-8.

26. Imaeda AB, Watanabe A, Sohail MA, et al. Acetaminophen-induced hepatotoxicity in mice is dependent on Tlr9 and the Nalp3 inflammasome. J Clin Invest 2009;119:305-14.

27. Martinon F. Dangerous liaisons: mitochondrial DNA meets the NLRP3 inflammasome. Immunity 2012;36:313-5.

28. Baroja-Mazo A, Martin-Sanchez F, Gomez AI, et al. The NLRP3 inflammasome is released as a particulate danger signal that amplifies the inflammatory response. Nat Immunol 2014;15:738-48.

29. Franklin BS, Bossaller L, De Nardo D, et al. The adaptor ASC has extracellular and 'prionoid' activities that propagate inflammation. Nat Immunol 2014;15:727-37.

30. Coll RC, Robertson AA, Chae JJ, et al. A small-molecule inhibitor of the NLRP3 inflammasome for the treatment of inflammatory diseases. Nat Med 2015;21:248-55. 